\title{
Online Traffic Condition Evaluation Method for Connected Vehicles Based on Multisource Data Fusion
}

\author{
Pang-wei Wang, ${ }^{1}$ Hong-bin Yu, ${ }^{1}$ Lin Xiao, ${ }^{2}$ and Li Wang ${ }^{1}$ \\ ${ }^{1}$ Beijing Key Lab of Urban Intelligent Traffic Control Technology, North China University of Technology, Beijing 100144, China \\ ${ }^{2}$ National Research Council of the National Academies of Sciences, Engineering, and Medicine, 500 Fifth Street NW, \\ Washington, DC 20001, USA \\ Correspondence should be addressed to Pang-wei Wang; wpw@ncut.edu.cn
}

Received 11 April 2017; Revised 4 June 2017; Accepted 2 July 2017; Published 3 August 2017

Academic Editor: Hai-Feng Ji

Copyright (C) 2017 Pang-wei Wang et al. This is an open access article distributed under the Creative Commons Attribution License, which permits unrestricted use, distribution, and reproduction in any medium, provided the original work is properly cited.

\begin{abstract}
With the development of connected vehicle (CV) and Vehicle to X (V2X) communication, more traffic data is being collected from the road network. In order to predict future traffic condition from connected vehicles' data in real-time, we present an online traffic condition evaluation model utilizing V2X communication. This model employs the Analytic Hierarchy Process (AHP) and the multilevel fuzzy set theory to fuse multiple sources of information for prediction. First, the contemporary vehicle data from the On Board Diagnostic (OBD) is fused with the static road data in the Road Side Unit (RSU). Then, the real-time traffic evaluation scores are calculated using the variable membership model. The real data collected by OBU in field test demonstrates the feasibility of the evaluation model. Compared with traditional evaluation systems, the proposed model can handle more types of data but demands less data transfer.
\end{abstract}

\section{Introduction}

Nowadays, traffic congestion is a serious issue due to the growing number of vehicles moving on the urban road networks. Connected Vehicle (CV) technology enhances the ability of traffic information collection and management through Vehicle to X communication (including Vehicle-ToInfrastructure (V2I) and Vehicle-To-Vehicle (V2V) communication), which presents one of the best ways to mitigate urban traffic congestion, improve traffic safety, and reduce fuel consumption [1].

With the development of connected vehicles, multiple sensors and communication modules tend to become standard equipment in vehicles. Through these sensors and communication modules, the required traffic information can be collected and distributed efficiently. Meanwhile, diversified traffic data sources and effective analysis methods provide a more reliable decision-making basis for traffic managers [2]. Furthermore, advanced data fusion technology can be used to deal with massive multisource traffic data to provide more accurate estimation of urban road conditions and improve the evaluation and prediction methods for urban traffic system [3].
In the urban traffic system, there are various traffic data acquisition methods, such as detector, video, and radar. With the application of V2X communication, more traffic data can be collected from connected vehicles, infrastructure, and other traffic sensors. The data can then be fed to the traffic condition evaluation, prediction, and decision-making system. If the traffic management department can leverage real-time traffic information from V2X communication to induce traffic flow and reduce unnecessary travel time, the operational efficiency of the transport network can be improved.

Huang proposed a data fusion method to optimize urban traffic flow based on neural network and fuzzy reasoning, which collected the traffic data from varied detectors on the urban road [4]. Quek et al. introduced a special class of fuzzy neural network known as the pseudo outer-product fuzzy neural network using the truth-value-restriction method (POPFNN-TVR) for short-term traffic flow prediction. The method combined the complementary capabilities of both neural networks and fuzzy logic; thus it constituted a more promising technique for modeling traffic flow [5]. Zhao et al. analyzed the characteristics of multisource data fusion and support vector machine (SVM). Following to the principle 
of SVM, they collected multisource traffic flow data from Hanshin Highway [6]. Castillo et al. reviewed the roles of mathematical tools and methods in traffic flow observability, estimation, and prediction problems. The high number of possible combinations of these elements justifies the existence of a wide collection of methods for analyzing static and dynamic situations [7]. Thomas and Dia presented a neural network algorithm based on traffic data fusion and tested it with simulated data. It analyzed various influence factors on data collection, such as positions of detectors, numbers of floating cars, length of the urban road, and severities of traffic accidents. Several classical algorithms were applied in traffic information fusion, including Kalman filter, artificial neural network, exponential smoothing, and recursive estimation algorithm [8]. Yang et al. proposed a novel fusion model which can be used to identify traffic status and analyze traffic conditions, accidents, scope of coverage, and forecast of future traffic flow [9]. Ren et al. processed observation traffic data for the traffic volume of urban road using fuzzy fusion algorithm. The test results showed that this method can acquire more complete and reliable traffic data. To forecast long periods of traffic flow conditions [10] Stutz and Runkler used fuzzy clustering to classify and analyze the traffic jams on a German freeway [11]. Jiang et al. used fuzzy clustering to identify road traffic conditions; the feasibility of this model was proved by the simulation results [12]. Rizzi et al. proposed an application of a highly efficient classification system based on low complexity real-time Internet traffic flows, by considering traffic data sets collected in different epochs and places [13]. Guo et al. proved the urban road traffic conditions can be analyzed with traffic data of coil detector by improved fuzzy clustering method [14]. He et al. improved a fusion method with new data collected from mobile phone and microwave sensors, providing enough data for traffic analysis [15].

There are several achievements while applying V2X communication in ITS. Backfrieder et al. predicted future congestion based on the Bottleneck prediction method and V2X communication. It demonstrated promised performance through dynamic microscopic traffic simulations both in a real-world scenario and in an artificial road network scenario [16]. Schünemann proposed a flexible simulation tool which simulated real-time traffic flow by V2X. This tool can also be used to simulate various scenarios of future intelligent transportation systems [17]. Otsuki and Miwa designed an efficient content-delivery control algorithm using real-time traffic data generated from traffic situation. The algorithm utilized the route prediction information in order to share traffic data during the vehicles by V2X communication efficiently [18]. Wedel et al. introduced a novel algorithm that can be used for connected vehicle with navigation system to calculate routes circumnavigating congested roads [19].

We can draw a conclusion from the literatures above that current research on traffic evaluation mainly focuses on how to process traditional data from detectors. Researchers have conducted a full study of how to improve the accuracy and reliability of information fusion. However, due to the limitation of data types and inevitable errors from traffic detectors, the advantages of novel traffic evaluation method are not prominent. To fill this research gap, a real-time traffic evaluation method based on data fusion in V2X scenario is presented in this paper. The vehicle OBD data is collected and processed by the RSU installed at the intersection. OBD data will be later fused with the static road data in the method. Details are discussed in the following section.

\section{Description of the Traffic Data Fusion Scenario}

Unlike traditional floating car system which sends real-time data directly to the server via mobile network and provides few data for traffic control, this paper uses the OBD data as the source of vehicle dynamic data and keeps the data within RSU at intersection. There are two types of data stored in the RSU: static road parameters such as road grade, number of lanes, road length, and real-time dynamic data generated by the connected vehicles.

The scenario of connected vehicles with V2X communication in this paper is described as shown in Figure 1. With the RSU installed at the intersection, all kinds of vehicle data generated by the vehicle passing through the road section (Point $\mathrm{A} \rightarrow$ Point $\mathrm{D}$ ) are collected. By fusing the parameters of the road section, the system evaluates the traffic conditions at each collection interval.

The structure of CV system is shown as Figure 2. The On Board Unit (OBU) installed on the vehicle is an embedded acquisition system that receives the vehicle data through the Controller Area Network (CAN) protocol from the Electronic Control Unit (ECU). The RSU actively sends the handshake information to establish communication with the OBU that supports V2X communication and determines whether the vehicle enters or leaves the intersection by comparing the location information of the intersection and the GPS data of the vehicle.

The flow chart of traffic data fusion is shown in Figure 3. When the vehicle leaves the last intersection (Point A), it starts to record the running data of the current road section and sends the data generated on the road section to the RSU when leaving the next intersection (Point D). When the vehicle enters the communication range (Point $\mathrm{B}$ ) of the RSU, the two parties will establish a stable V2X communication. The OBU sends its own vehicle basic information and continuously sends the positioning information before the RSU requests the OBD data. When the vehicle enters the intersection 2 (Point $\mathrm{C}$ ), since the communication history of the RSU1 is recorded in the OBU, the current vehicle data belonging to entrance lane (intersection $1 \rightarrow$ intersection 2) can be determined in the RSU2. According to this principle, the data of vehicles on multiple entrance lanes can be processed simultaneously in the RSU. After the data sent by the $\mathrm{OBU}$ is verified as valid, data fusion and evaluation will be done in the RSU according to the model in Section 3.

The OBD interface is chosen since it provides not only the vehicle sensor information, but also the vehicle internal control information and fault information. This distinguishes the proposed model from most traditional floating car data collection systems which obtain the vehicle data mainly from GPS module. Since the OBD interface integrates external 


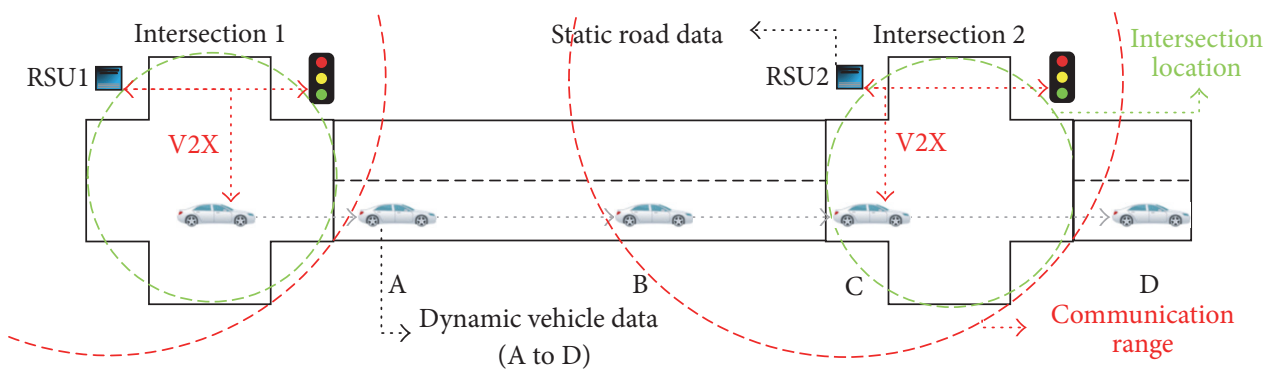

FIgURE 1: The scenario of CV system on intersections.

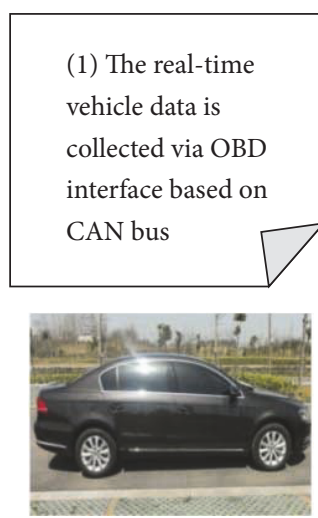

Experimental Vehicle

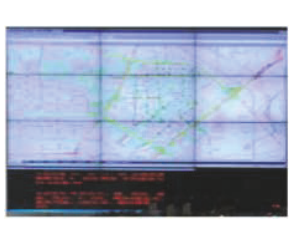

Remote

Server

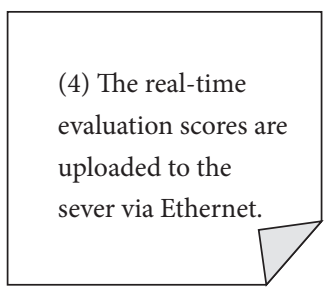

FIgure 2: The evaluation process for CV system.

detectors, it greatly enhances the evaluation system's versatility.

With the V2X communication and vehicle OBD data, the scenarios described in this paper have the following advantages over traditional floating car systems:

(1) More traffic information is shared by V2X. The traffic flow data on the macroscale is fused and calculated at the $\mathrm{RSU}$, whereas the running information of each vehicle is collected in V2X communication on the microscale.

Meanwhile, the information for driving guidance or alarm can be sent to vehicles as well. In a word, if the

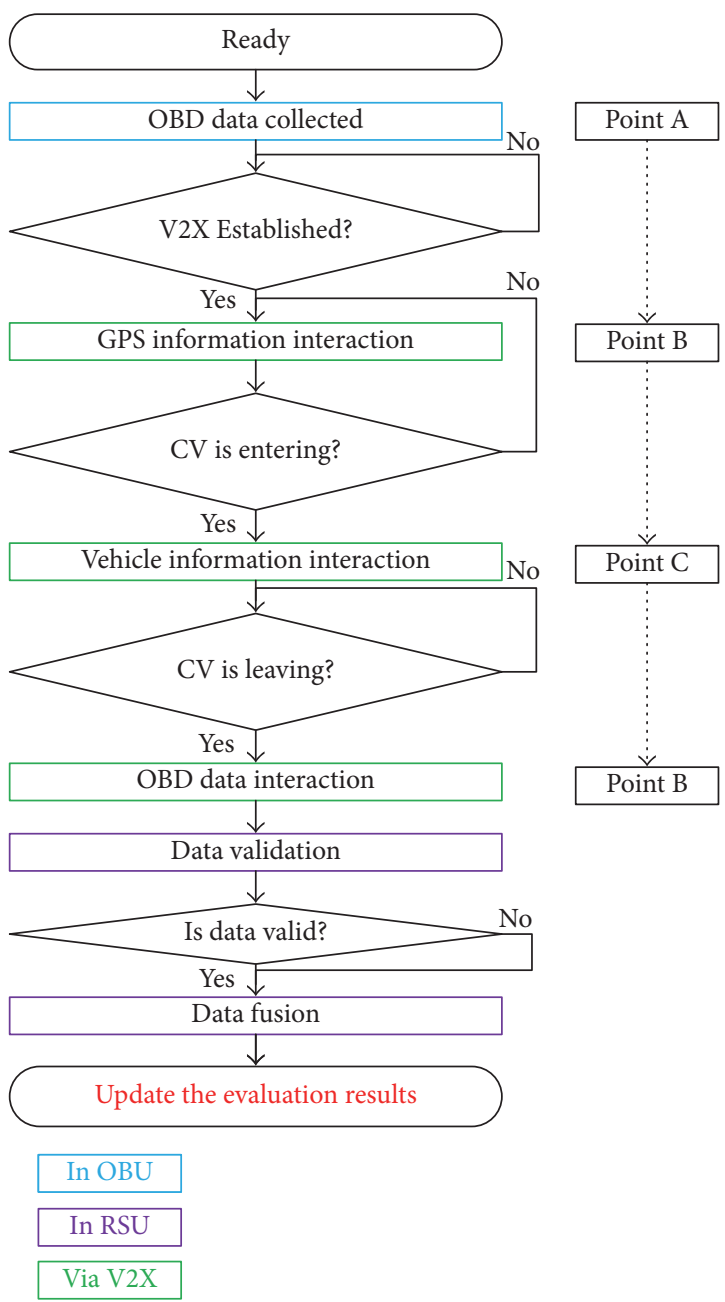

Figure 3: The flow chart of online traffic evaluation based on data fusion with V2X.

multisource data fusion with V2X communication is applied in urban traffic system, it can greatly improve the driving safety and traffic capacity. The accuracy error caused by the interference of the sensor can also be avoided.

(2) Traffic data from OBD interface is more accurate and computationally friendly. The method reduces the load and the computation amount of the data on the network and can effectively avoid errors caused by the collection system. Since the method collects corresponding dynamic data judged 


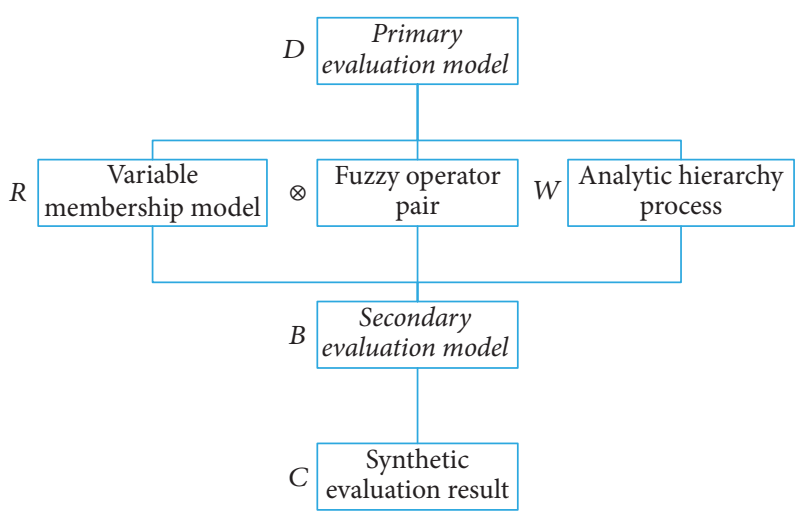

FIgURE 4: The structure of evaluation model.

based on the OBD data directly, it is not necessary to always track and calculate the vehicle GPS data. The process of data calculation and transmission is simplified by the method, and errors caused by sensors can also be avoided.

\section{Evaluation Model Based on Real-Time Traffic Information Fusion}

The evaluation model established in this paper is based on the multilevel fuzzy synthetic evaluation model. The basic idea is to establish the fuzzy judgment matrix by using the transformation principle to describe the data boundary of the factors in fuzzy set. Through the multilayer numerical calculation based on the evaluation criteria and weights, we will determine the results of the evaluation object [20,21]. The structure of synthetic evaluation model is shown as Figure 4.

3.1. The Primary Evaluation Model. Define $Q$ as a finite set, and $Q: Q=\left\{q_{1}, q_{2}, \ldots, q_{n}\right\}$, where $q_{i}(i=1,2, \ldots, n)$ is an object to be evaluated.

The objects to be evaluated (elements of " $Q$ ") in this paper are the road states at different times (e.g., 10 a.m. or 5 p.m.).

Define a finite set, that is, $P: P=\left\{p_{1}, p_{2}, \ldots, p_{m}\right\}$, and the element $p_{i}(i=1,2, \ldots, m)$ in $P$ represents a different evaluation indices.

While evaluating a road's real-time status, there are many evaluation indexes that can be used. Based on the principle of measurability, the average travel time (ATT), average number of stops (ANS), and average stopped time (AST) are selected as the final evaluation indices in this model, as in (1). All these three indices can be calculated from data collected from OBD and V2X communication.

$$
P=\left\{p_{1}, p_{2}, p_{3}\right\}=\{\text { ATT, ANS, AST }\} .
$$

Travel time is defined as the difference between the data exchange time at the current intersection and that at the adjacent downstream intersection. While traveling to the next intersection, the OBD can record the number of stops and total stopped time. In addition, all detected data will be verified by communication integrity and data validation to ensure the validity of the data. Then, the above selected three real-time evaluation indices can be calculated by averaging the valid data.

Define $u_{j}$ as the membership function of the $j^{\text {th }}$ evaluation index $p_{j} \in P$, that is, $u_{j}=u\left(p_{j}\right), u_{j} \in[0,1]$; then $U$ is a finite fuzzy subset; that is, $U=\left\{u_{1}, u_{2}, \ldots, u_{m}\right\}$.

Since the membership function is applicable to all evaluation objects, an evaluation matrix can be obtained as a fuzzy relation, that is, $R: Q \times U \rightarrow[0,1]$, which is defined as

$$
R=\left[\begin{array}{c}
R_{1} \\
R_{2} \\
\vdots \\
R_{n}
\end{array}\right]=\left[\begin{array}{cccc}
r_{11} & r_{12} & \cdots & r_{1 m} \\
r_{21} & r_{21} & \cdots & r_{2 m} \\
\vdots & \vdots & \ddots & \vdots \\
r_{n 1} & r_{n 2} & \cdots & r_{n m}
\end{array}\right]_{n \times m},
$$

where $r_{i j}=R\left(q_{i}, u_{j}\right) \in[0,1]$ is the membership degree of the $i^{\text {th }}$ object to be evaluated on the $j^{\text {th }}$ evaluation index.

Define $S=(Q, U, R)$ as the primary evaluation space and give a fuzzy vector $W$ :

$$
W^{\prime}=\left(w_{1}^{\prime}, w_{2}^{\prime}, \ldots, w_{m}^{\prime}\right)^{T} .
$$

In (3), element $w_{j}$ of $W$ represents the weight of each evaluation index with respect to the primary evaluation model:

$$
D=R \otimes W
$$

3.2. The Variable Membership Model. Assuming that the evaluation result is a finite set $V=\left\{v_{1}, v_{2}, v_{3}, v_{4}\right\}=$ \{excellent, good, medium, bad\}, each element in the set corresponds to a distribution interval of the membership function, which is shown in

$$
\begin{gathered}
u_{j} \in[0.25(i-1), 0.25 i], \\
v=v_{i} \quad(i=1,2,3,4) .
\end{gathered}
$$

Considering the negative correlation between the evaluation indices and evaluation results, this paper selects the membership function of the Cauchy type, as shown in

$$
\begin{aligned}
& u_{i}=\mu\left(p_{j}\right)= \begin{cases}1, & p_{j} \leq c_{j} \\
\frac{1}{1+\left[a_{j}\left(p_{j}-c_{j}\right)\right]^{b_{j}}}, & p_{j}>c_{j}\end{cases} \\
& \forall a_{j}, b_{j}, c_{j}>0 .
\end{aligned}
$$

It can be seen that, in the coordinate system $p_{j}-u_{j}$, each membership function distribution interval $0.25 i$ must have a corresponding critical value $p_{i j}$. According to the set of values under the same membership function, the coefficients of the membership function, including $a_{j}, b_{j}$, and $c_{j}$, can be solved by regression analysis. Taking into account the actual traffic scenario, $p_{i j}$ is a time-varying value changing with static traffic parameters. The dynamic adjustment strategy for $p_{i j}$ is shown in

$$
p_{i j}=\frac{(1-\xi) \bar{p}_{i j} L \omega_{m j}}{\widehat{L} \alpha_{n j}}+\xi \bar{p}_{i j}, \quad(i=1,2,3,4),
$$


TABLE 1: Parameters of the membership function.

\begin{tabular}{cccccccc}
\hline & $\bar{p}_{i j}$ & $\xi$ & $\omega_{m j}$ & $\alpha_{n j}$ & $p_{i j}$ & $a_{j}$ & $b_{j}$ \\
\hline$u_{1}$ & $(184,138,97,71)$ & 0.3 & 1 & 1.05 & $(219,165,117,86)$ & 0.0007 & 1.66 \\
$u_{2}$ & $(3.8,2.6,1.6,0.9)$ & 0 & 0.95 & 1.10 & $(5.5,3.8,2.3,1.2)$ & 0.164 & 1.82 \\
$u_{3}$ & $(75,50,35,26)$ & 0.85 & 0.95 & 1.03 & $(80,54,37,28)$ & 0.165 & 1.29 \\
\hline
\end{tabular}

where $\bar{p}_{i j}$ is a typical critical value for the length of the unidirectional road at the specified road grade and is calibrated by a large number of tests, $\xi$ is a coefficient that represents the part of evaluation indices which is generated by the control of the signal, which depends mainly on the green signal ratio and the number of phases, $\bar{L}$ is the standard length $(500 \mathrm{~m})$ for the urban road, $\alpha_{n j}$ is the influence coefficient of $n$ lanes on the $j^{\text {th }}$ evaluation index, and $\omega_{m j}$ is the influence coefficient of $m$ branch road on the $j^{\text {th }}$ evaluation index.

To obtain the critical value $p_{i j}$, we take the relevant static data (in Table 4) and typical values into (7). By the nonlinear regression analysis function "nlinfit" in MATLAB, we obtain the parameters of the Cauchy-type function in (6). Then the coefficients are fitted as a curve, including $a_{j}, b_{j}$, and $c_{j}$. The results are shown in Table 1 and Figure 5.

For the value of the membership function $u_{j}$, it is necessary to convert it into the membership degree with the corresponding indices: based on the trapezoidal membership, we define the interval as $0.25 i$ of the evaluation result set $V$, which is the intermediate membership degree $\left(r_{i j}=0.5\right)$ of the two evaluation indices. The floating range is from $0.25 i-$ 0.1 to $0.25 i+0.1$. The final membership relationship is shown in Figure 6.

3.3. The Analytic Hierarchy Process. The Analytic Hierarchy Process (AHP) is used for organizing and analyzing complex decisions based on mathematics and psychology. Rather than prescribing a "correct" decision, the AHP helps decision makers find one solution that best suits their goal and their understanding of the problem. It provides a comprehensive and rational framework for structuring a decision problem, for representing and quantifying its elements, for relating those elements to overall goals, and for evaluating alternative solutions.

In the proposed model, there are many evaluation indexes that can be considered. However, the weights of each index are not predefined. For example, some researchers may regard that the average speed is the most important index to evaluate a road state, but others may regard the stops as more important than the average speed. Both of the two viewpoints are subjective assumptions. So the AHP method is to determine the weight of these evaluation indexes scientifically. The steps of AHP are shown as follows.

Step 1. According to the relevant research and practical experience, comparing the importance of the three evaluation indices, the judgment matrix table can be acquired as shown in Table 2.

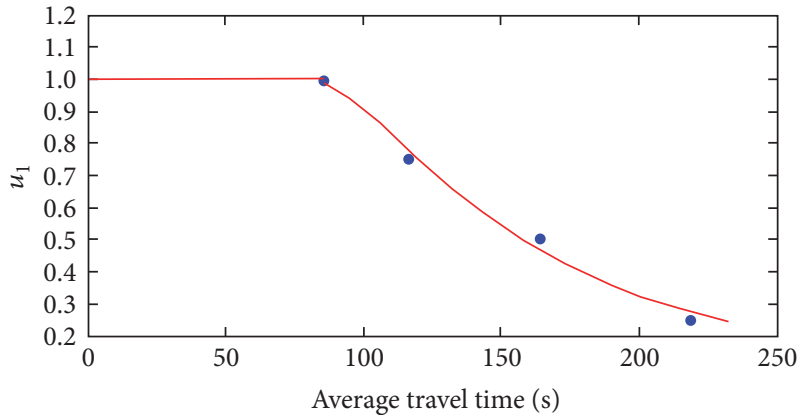

(a)

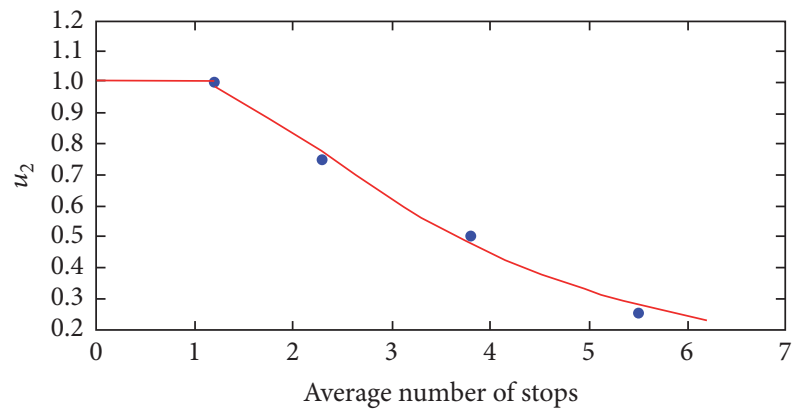

(b)

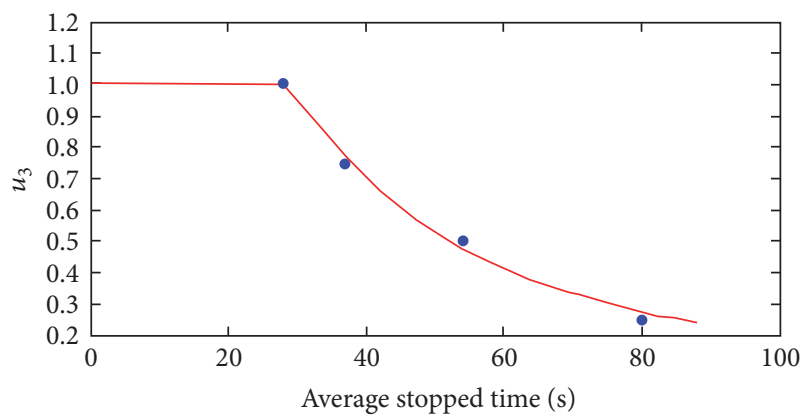

(c)

FIGURE 5: Curve fitting of the membership function.

Step 2. The data in Table 2 is brought into the following equation to obtain the judgment matrix $A$ :

$$
A=\left[\begin{array}{c}
a_{1} \\
a_{2} \\
\vdots \\
a_{n}
\end{array}\right]=\left[\begin{array}{cccc}
a_{11} & a_{12} & \cdots & a_{1 n} \\
a_{21} & a_{21} & \cdots & a_{2 n} \\
\vdots & \vdots & \ddots & \vdots \\
a_{n 1} & a_{n 2} & \cdots & a_{n n}
\end{array}\right]_{n \times n} .
$$




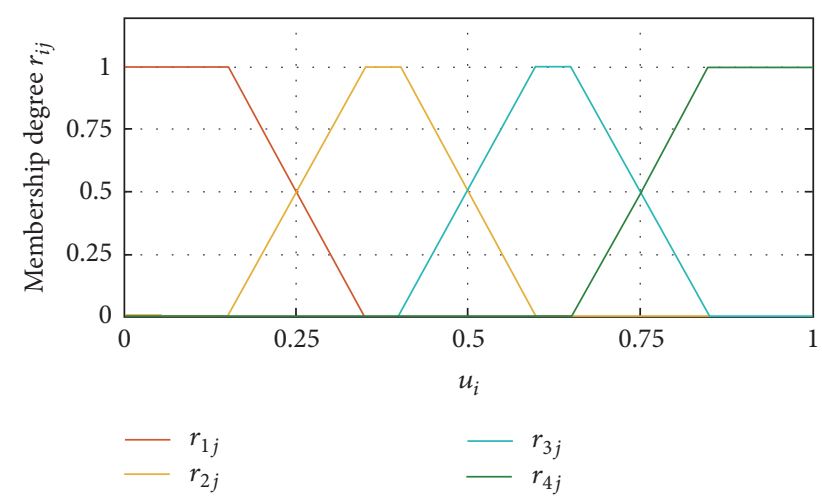

FIGURE 6: Membership relationship of the evaluation model.

TABLE 2: Parameters of the judgment matrix.

\begin{tabular}{llcc}
\hline & $a_{1}$ & $a_{2}$ & $a_{3}$ \\
\hline$a_{1}$ & 1 & 3 & 2 \\
$a_{2}$ & $\frac{1}{3}$ & 1 & $\frac{2}{3}$ \\
$a_{3}$ & $\frac{1}{2}$ & $\frac{3}{2}$ & 1 \\
\hline
\end{tabular}

Step 3. The columns of judgment matrix $A$ are normalized as

$$
\bar{a}_{i j}=\frac{a_{i j}}{\sum_{k=1}^{n} a_{k j}} \quad(i, j=1,2, \ldots, n) .
$$

To apply the AHP method, each element in (9) is recalculated as a proportion to the sum of its own column. By normalizing, the value of each element can be transformed as a percentage, which is the value that needs to be calculated in (10)

Step 4. The sum of the rows of the judgment matrix $A$ is calculated as

$$
\bar{w}_{i}=\sum_{j=1}^{n} \bar{a}_{i j} \quad(i=1,2, \ldots, n) .
$$

Step 5. Normalize $\bar{w}_{i}$ to get $w_{i}$; we can find the largest eigenvalue $\lambda_{\max }$ and eigenvector, according to $A w=\lambda_{\max } w$ :

$$
w_{i}=\frac{\bar{w}_{i}}{\sum_{i=1}^{n} \bar{w}_{i}} \quad(i=1,2, \ldots, n)
$$

Finally, the weight matrix can be calculated: $W=(0.52$, $0.22,0.26)^{T}$.

When the weight set is calculated by AHP method, we will make a consistency check to ensure that the results are reasonable. For example, to avoid logical errors, if the result is index $A$ being more important than index $B$, and index $B$ is more important than index $C$, but index $C$ is more important than index $A$, then the results are unreasonable.
Step 6. Calculate the consistence index C.I; we can find the corresponding mean random consistency index R.I, where $n$ represents the order of the judgment matrix $A$ :

$$
\text { C.I }=\frac{\lambda_{\max }-n}{n-1} .
$$

Step 7. The consistency ratio C.R is calculated as (13), where R.I represents a constant value, determined by $n$ (e.g., $n=$ 3 , R.I $=0.52$ ):

$$
\text { C.R }=\frac{\text { C.I }}{\text { R.I }} .
$$

Through the calculation in (13), the results of consistency check $(C . R<0.1)$ are accepted.

3.4. The Fuzzy Operator Pair and Secondary Evaluation Model. The symbol $\otimes$ in (4) represents a fuzzy operator pair. If more operator pairs are introduced at the same time, a new fuzzy subset can be obtained for each evaluation object:

$$
U^{\prime}=\left\{D_{1}, D_{2}, \ldots, D_{p}\right\}, \quad U^{\prime} \in[0,1] .
$$

In (14), $p$ represents the number of fuzzy operator pairs.

The fuzzy operator pair will determine the meaning of the fuzzy vector to a larger extent. Besides, the secondary evaluation space composed of multiple operator pairs will help to measure the influence of the evaluation indices $(P)$ on the object to be evaluated $(Q)$ from various aspects. In this paper, we select three operator pairs: $(\Lambda, \vee),(\bullet, \vee)$, and $(\wedge, \oplus)$, where " $\vee$ " represents Max, " $\wedge$ " represents Min, “॰" represents multiplication, and " $\oplus$ " represents addition. These three operator pairs focus on the contribution of individual or multiple evaluation indexes, and $\sum_{j=1}^{p} w_{j} \neq 1$.

A new fuzzy relation can be obtained Combining $Q$ and $U^{\prime}$, that is, $R^{\prime}: Q \times U^{\prime} \rightarrow[0,1]$ :

$$
R^{\prime}=\left[\begin{array}{c}
R_{1}^{\prime} \\
R_{2}^{\prime} \\
\vdots \\
R_{n}^{\prime}
\end{array}\right]=\left[\begin{array}{cccc}
d_{11} & d_{12} & \cdots & d_{1 p} \\
d_{21} & d_{21} & \cdots & d_{2 p} \\
\vdots & \vdots & \ddots & \vdots \\
d_{n 1} & d_{n 2} & \cdots & d_{n p}
\end{array}\right]_{n \times p} .
$$

In (15), $d_{i j}$ represents the primary evaluation value of the $i^{\text {th }}$ object calculated from (4) when the $j^{\text {th }}$ operator pair is used.

Thus, the secondary evaluation space $S^{\prime}=\left(Q, U^{\prime}, R^{\prime}\right)$ is obtained. Each element $w_{j} \in W$ is given a fuzzy vector $W^{\prime}$ in the secondary evaluation space:

$$
W^{\prime}=\left(w_{1}^{\prime}, w_{2}^{\prime}, \ldots, w_{p}^{\prime}\right) .
$$

In (16), the element $w_{j}^{\prime}$ of $W^{\prime}$ represents the weight of the $j^{\text {th }}$ fuzzy operator pair for the secondary evaluation space, and $\sum_{j=1}^{p} w_{j}^{\prime} \neq 1, w_{j}^{\prime} \in[0,1]$.

The secondary fuzzy vector matrix is obtained according to the AHP, that is, $W^{\prime}=(0.17,0.28,0.55)$. Then the secondary evaluation model is obtained:

$$
B=W^{\prime} R^{\prime T}=\left\{b_{1}, b_{2}, \ldots, b_{m}\right\} .
$$


TABLE 3: Data transmission performance with respect to communication distance.

\begin{tabular}{lcccc}
\hline Number & Communication distance $(\mathrm{m})$ & Packet loss rate $(\%)$ & Time delay $(\mathrm{ms})$ & Transmission rate $(\mathrm{kbps})$ \\
\hline 1 & 20 & 1.6 & $<200$ & 102.2 \\
2 & 50 & 1.9 & $<200$ & 99.5 \\
3 & 100 & 3.3 & $<400$ & 96.5 \\
4 & 150 & 5.6 & $<400$ & 91.9 \\
5 & 200 & 7.2 & $<800$ & 87.3 \\
6 & 300 & 9.9 & $<800$ & 80.5 \\
7 & 400 & 21.7 & $>1200$ & 71.5 \\
8 & 500 & 46.6 & $>1200$ & 50.4 \\
\hline
\end{tabular}

Note. The test data consists of three packets, totaling $51 \mathrm{~KB}$.

In (17), $b_{i}$ represents the evaluation index of the $j^{\text {th }}$ evaluation object, where $b_{i}=\sum_{j=1}^{p} w_{j}^{\prime} d_{i j}$.

We can find that this mathematical model is a two-level fuzzy evaluation model. The final result is a set of evaluation results.

3.5. The Synthetic Evaluation Results. Finally, the evaluation results for connected vehicles are obtained based on the synthetic evaluation method. To ensure the feasibility, the original evaluation results $B$ are integrated according to the weighted average principle. Firstly, the elements of $B$ are normalized to obtain $\widetilde{b}_{i}$; then the final evaluation score $C$ is calculated in

$$
C=100-\sum_{i=1}^{m} \frac{100}{3}(i-1) \widetilde{b}_{i} .
$$

We can find in (18), where $C \in[0,100]$, that the road condition is proportional to the value of $C$.

\section{Experiment and Analysis}

4.1. Experimental Method. A data acquisition system with reference to a real V2X communication scenario is established in this article. The system has an embedded data acquisition device based on the chip of Freescale i.MX6 Q, which is installed in vehicles (OBU) and intersections (RSU). The device has a rich interface as shown in Figure 7, and the data of the Experimental vehicle can be passed into the device via the CAN interface. The OBU acquires OBD and GPS data according to the implementation procedure and communicates with RSU through the high power Zig-Bee module. The actual environment proved that the communication established by the Zig-Bee module was stable enough to simulate the real V2X communication. The test results are shown in Table 3.

A segment of the Pingguoyuan South Road in Shijingshan District of Beijing is picked as experimental section in this paper. The static data of this road is shown in Table 4. In the experiment, two experimental vehicles equipped with OBU device are traveling continuously on both sides of the road. The calculated evaluation indices are transmitted via wireless communication to the RSU device installed at the
TABLE 4: Parameters of the road section.

\begin{tabular}{lc}
\hline Parameter name & Data \\
\hline Road grade & Secondary trunk road \\
Road type & Two-way two-lane \\
Road length & $698 \mathrm{~m}$ \\
The number of branch road & $1 / 1$ \\
Traffic signal control & Yes \\
\hline
\end{tabular}

intersection. The experiment is carried out from 6:00 am to 8:00 pm on March 3, 2017. In order to improve the accuracy of the evaluation as much as possible, the experiment ensured that there are at least 10/16 sets of data in each section of the road during the peak/valley hours of traffic flow. At the same time, the flow data of the day is obtained through artificial observation and converted to Passenger Car Unit (PCU). Restricted by the experimental condition, the final evaluation result is calculated by deriving the vehicle data stored in the RSU into the personal computer. However, this does not affect the evaluation result and preserves more details of the original data to ensure the reliability of the results.

4.2. Experimental Data Analysis. A total of 407 data packets were collected by the experimental device, of which 377 were valid. That is to say, $92.6 \%$ of all data packets received by the device is valid. All valid data are processed at intervals of one hour as shown in Figures 8, 9, and 10 where (a) represents a section from west to east, (b) represents a section from east to west, the curve represents the three evaluation indices, and the gray shaded area represents the distribution of the data. As seen from the figures, the three evaluation indices share similar trend with respect to time. This proves the reliability of the data from one aspect.

The data sheets of the three evaluation indices are brought into a MATLAB program where the final synthetic evaluation result $C$ is calculated; see the lower part of Figure 11. The two colors of the data represent the two directions of the road. The upper part of Figure 10 is the flow data for the day. Comparing the upper and lower parts of the figure, we can find that the evaluation score has a negative correlation with the original traffic flow data, which is consistent with the actual traffic situation. As shown in the figure, the peak flow in the day also 


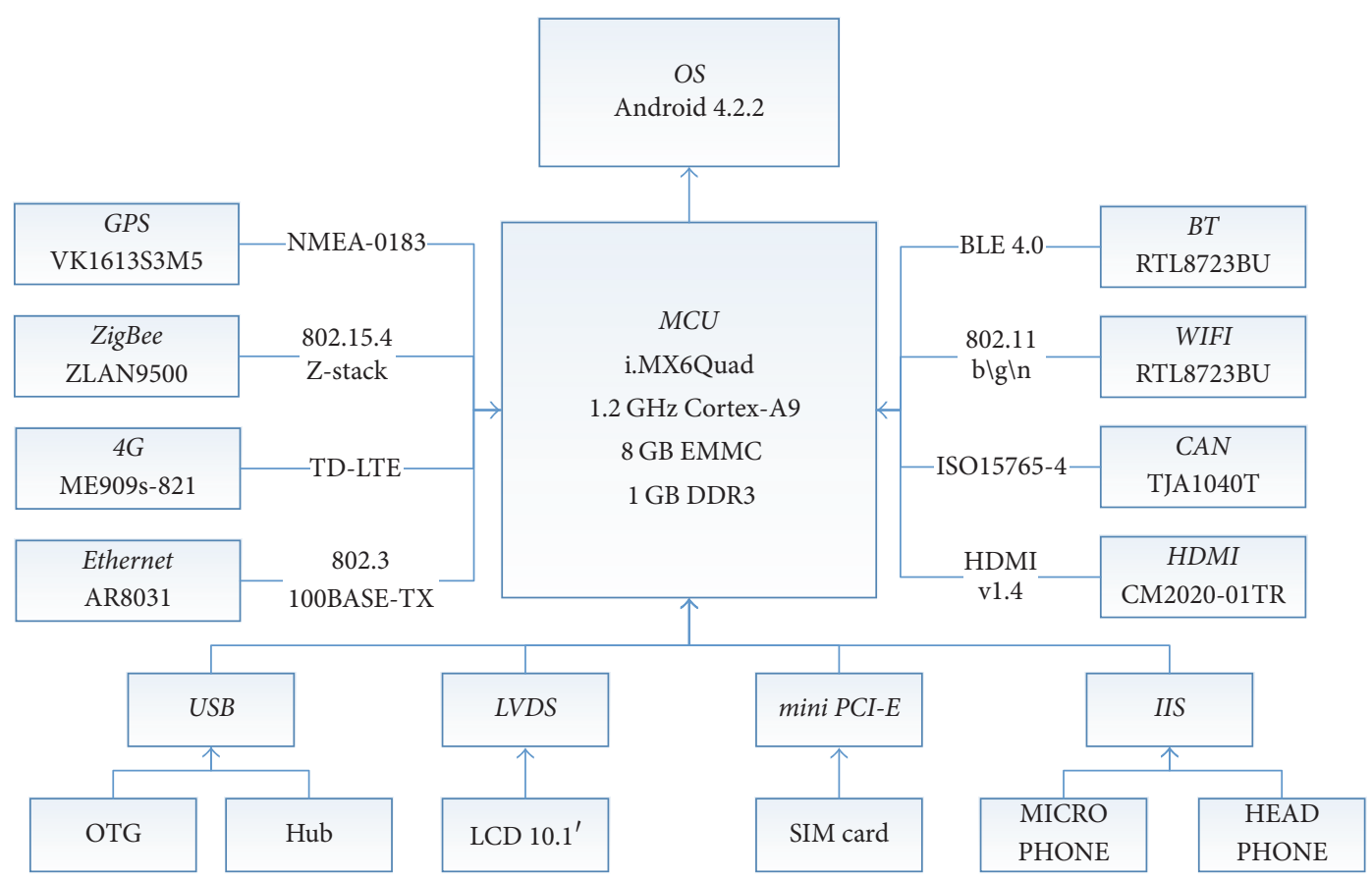

FIgURE 7: The hardware structure of the terminal for CV system.

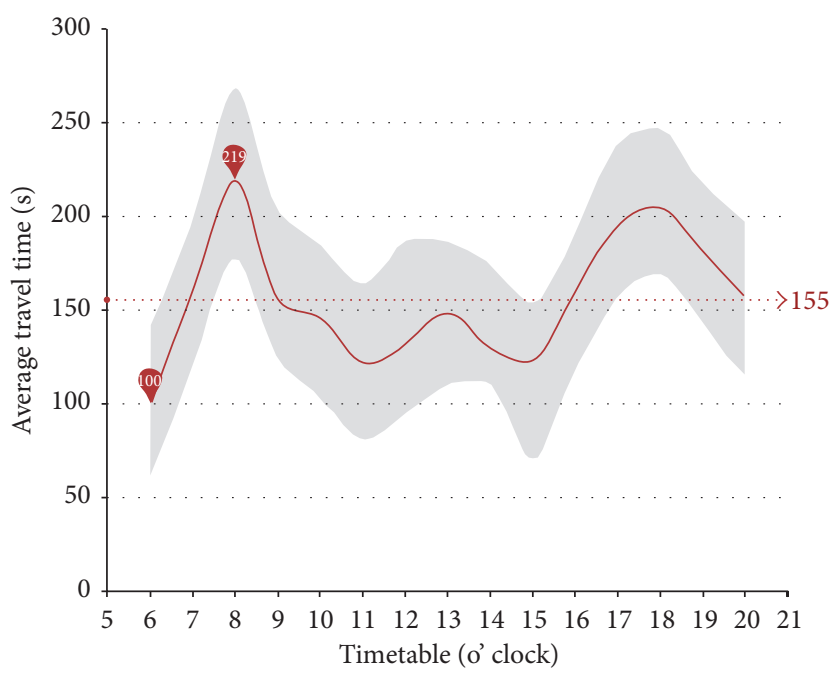

(a) West to east

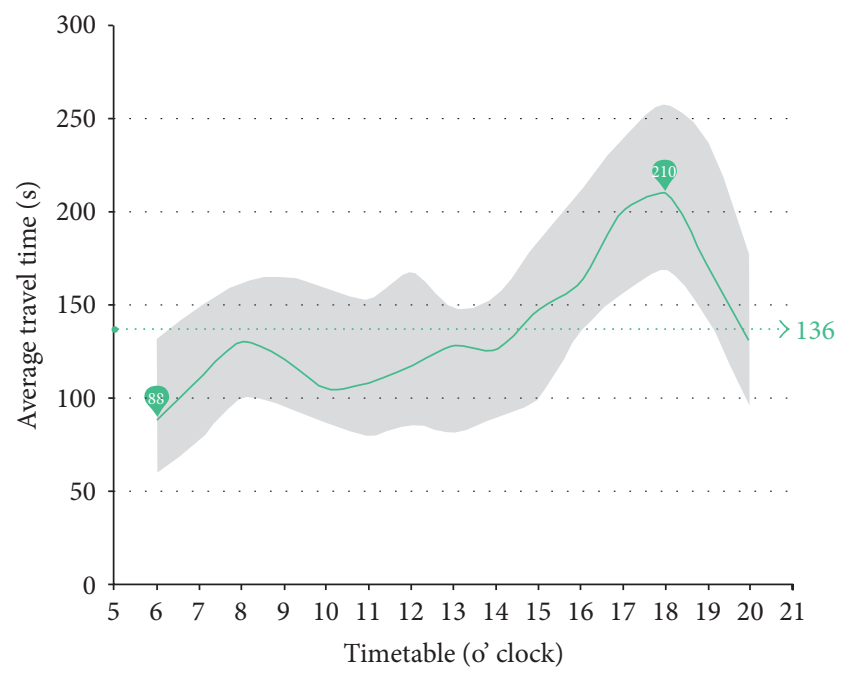

(b) East to west

FIGURE 8: Results of average travel time.

corresponds to the lowest evaluation result. The difference between the peak hours and the valley hours is evident in the evaluation results.

Since the two directions of the road have similar static road parameters, in order to describe the relationship correctly between the evaluation score and the traffic flow, all the evaluation data are arranged in ascending order of the traffic flow as shown in Figure 12. The red line in the figure represents the actual calculated evaluation score, and the green line represents the reference delay value of the grade road at different traffic flows.

It can be seen from the above analysis that the evaluation results of the multilevel fuzzy synthetic method used in this paper are in good agreement with the actual situation. If the device's communication coverage is further improved, the time interval of the traffic evaluation can be reduced to 5 to 15 minutes, which is sufficient to meet the requirements of the road evaluation system under the complex road network. It 


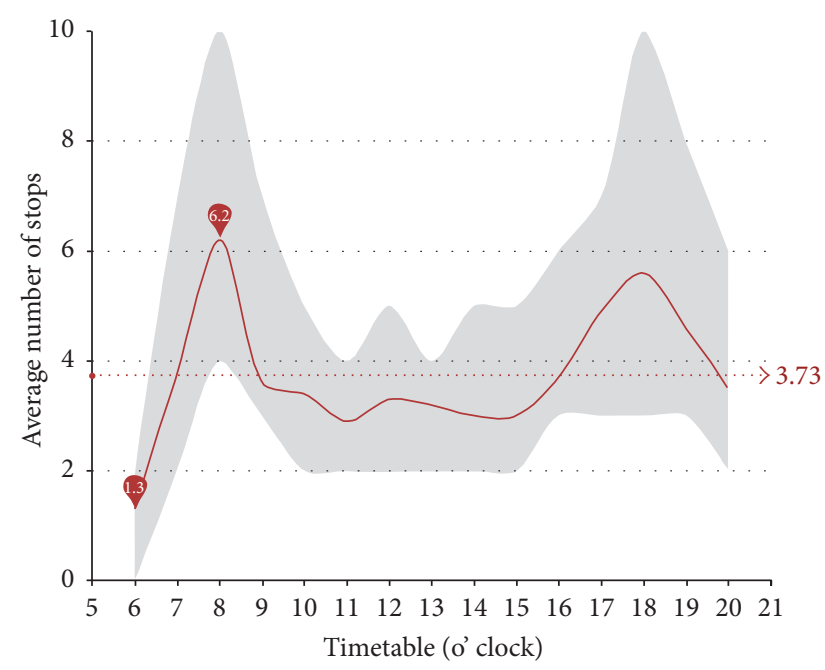

(a) West to east

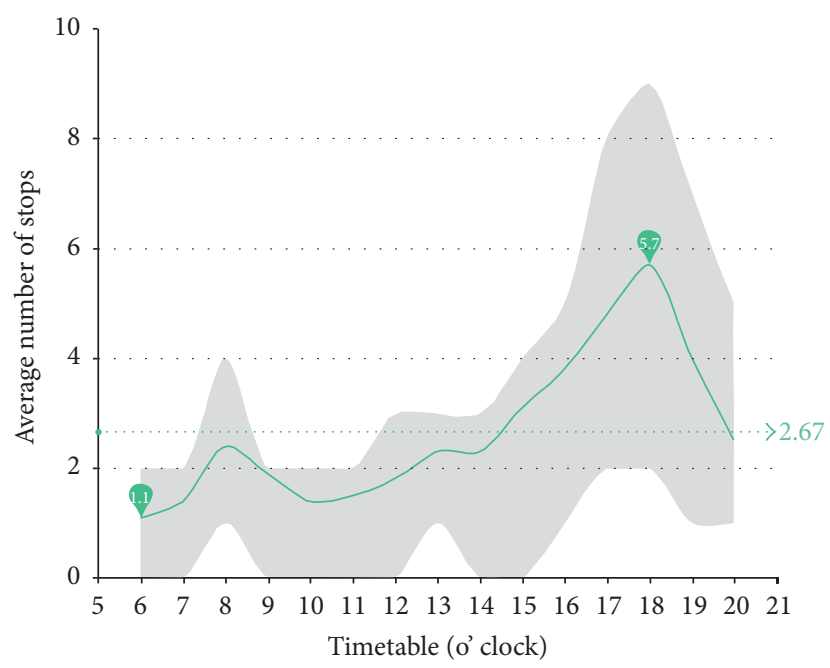

(b) East to west

FIGURE 9: Results of average stops.

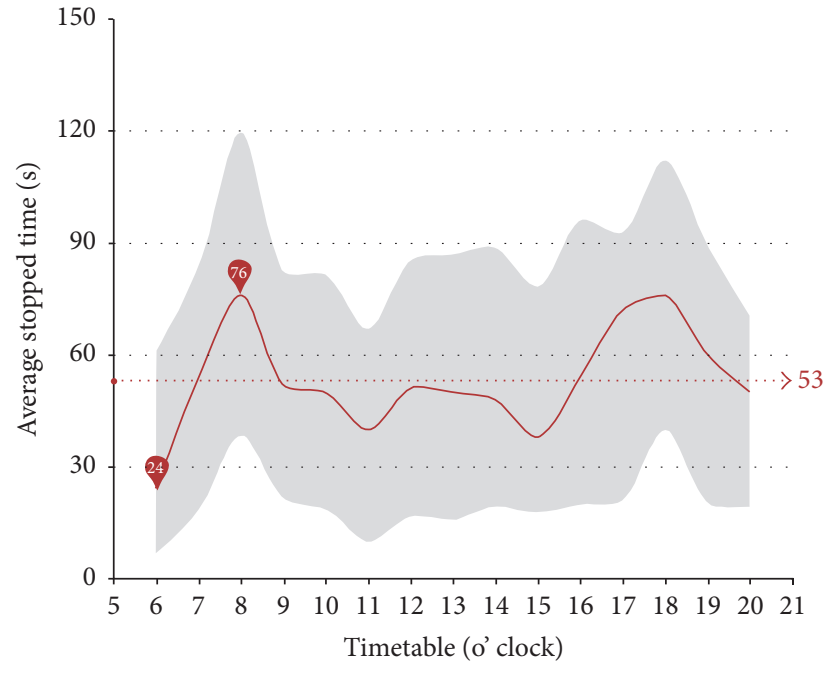

(a) West to east

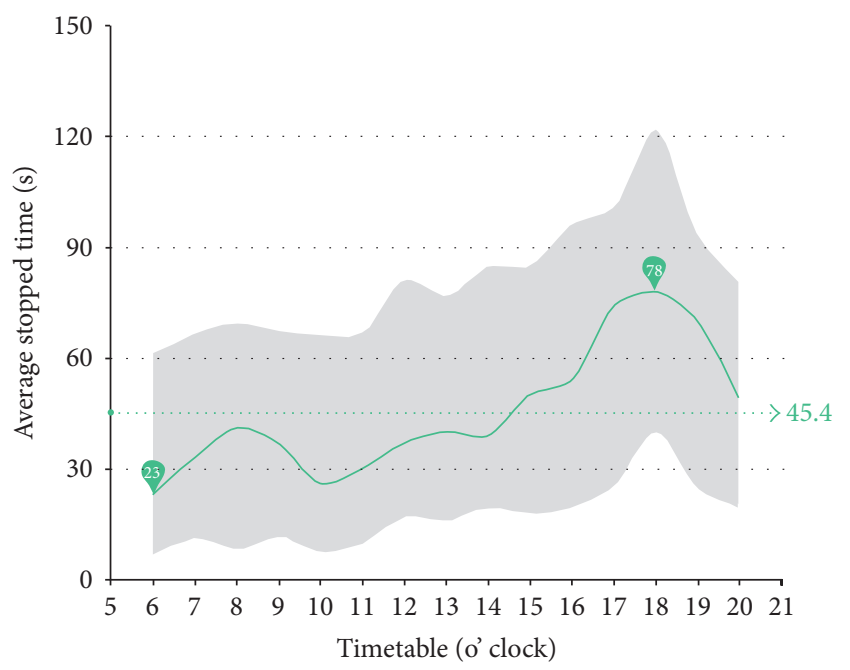

(b) East to west

FIGURE 10: Results of average stopped time.

provides a feasible solution for the traffic evaluation method under the V2X scenario.

\section{Conclusion}

By fusing the real-time connected vehicle data with static road segment information, an online traffic condition estimation model is proposed and tested in this paper. The OBD data and the traffic evaluation method are applied in the system of connected vehicles on urban road with V2X. Based on the traditional fuzzy synthetic model, the multioperator synthetic fuzzy and variable membership model is introduced. We determined the scientific model parameters through AHP. In the field experiment, the evaluation results produced by the proposed model are the same with the actual situation of the road, which demonstrates the fidelity and effectiveness of the method. When the OBD data in the vehicle is collected by the V2X, the proposed model has greater advantage over the traditional floating vehicle data evaluation method. In addition, since connected vehicles are supplied with more detailed traffic information, the traffic capacity and safety can be greatly improved in the foreseeable future.

\section{Disclosure}

The views are those of the authors alone.

\section{Conflicts of Interest}

The authors declare that they have no conflicts of interest. 


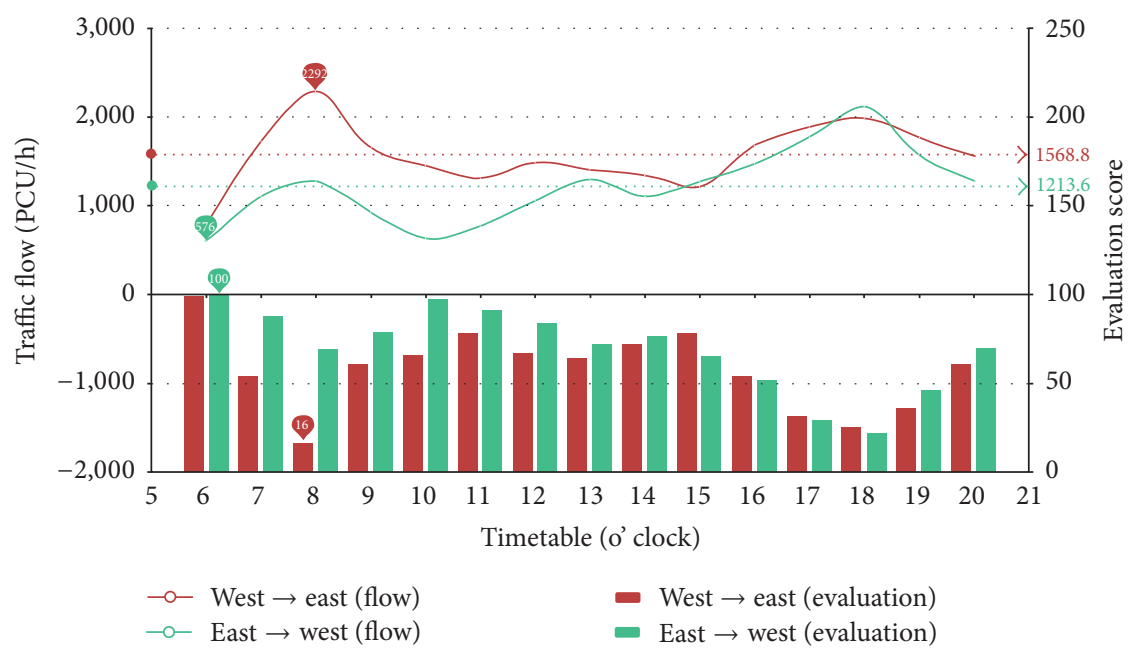

FIgURE 11: Comparison of evaluation scores and traffic conditions (traffic flows) for connected vehicles.

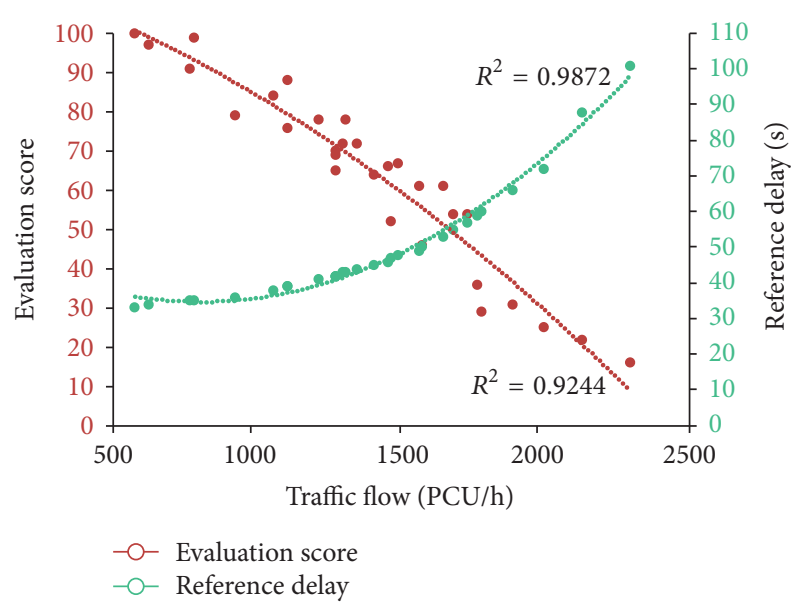

FIgURE 12: Comparison of evaluation scores and traffic conditions (reference delay) for connected vehicles.

\section{Acknowledgments}

This research was supported by grants from the Beijing Natural Science Foundation (4174088), National Natural Science Foundation of China (61603004), and the Scientific Research Project of Beijing Education Committee (Grant nos. PXM2017-014212-000031, PXM2017-014212-000033).

\section{References}

[1] J. Hu, B. B. Park, and A. E. Parkany, “Transit signal priority with connected vehicle technology," Transportation Research Record Journal of the Transportation Research Board, vol. 2418, pp. 20 29, 2014.

[2] J. Hu, B. B. Park, and Y.-J. Lee, "Coordinated transit signal priority supporting transit progression under Connected Vehicle Technology," Transportation Research Part C: Emerging Technologies, vol. 55, pp. 393-408, 2015.
[3] C. Ding, D. G. Wang, C. Liu, Y. Zhang, and J. W. Yang, "Exploring the influence of built environment on travel mode choice considering the mediating effects of car ownership and travel distance," Transportation Research Part A: Policy and Practice, vol. 100, pp. 65-80, 2017.

[4] Q. L. Huang, "Data Fusion of multi-detector in the same traffic detecting section based on NN-FR," Journal of Highway and Transportation Research and Development, vol. 23, no. 7, pp.120$124,2006$.

[5] C. Quek, M. Pasquier, and B. B. S. Lim, "Pop-traffic: A novel fuzzy neural approach to road traffic analysis and prediction," IEEE Transactions on Intelligent Transportation Systems, vol. 7, no. 2, pp. 133-146, 2006.

[6] N. L. Zhao, L. Yu, Y. B. Geng, and X. M. Chen, "Support Vector Machine-Based Approach to Data-Layer Multi-Source ITS Data Fusion," Journal of Transportation Systems Engineering and Information Technology, vol. 7, no. 2, pp. 32-37, 2007.

[7] E. Castillo, Z. Grande, A. Calviño, W. Y. Szeto, and H. K. Lo, "A State-of-The-Art Review of the Sensor Location, Flow Observability, Estimation, and Prediction Problems in Traffic Networks," Journal of Sensors, vol. 2015, Article ID 903563, 2015.

[8] K. Thomas and H. Dia, "A neural network model for arterial incident detection using probe vehicle and loop detector data," in Conference of Australian Institutes of Transport Research (CATIT '2000), pp. 6-8, Australian National University, Canberra, Australia, 2000.

[9] Z. S. Yang, S. Wang, and D. S. Ma, "Review of basic traffic data fusion methods," Journal of Highway and Transportation Research and Development, vol. 23, no. 3, pp. 111-116, 2006.

[10] Y. W. Ren, J. Lu, H. Y. Wang, and Q. J. Xiang, "Fuzzy fusion arithmetic for adjusting observed traffic data," Journal of Highway and Transportation Research and Development, vol. 23, no. 9, pp. 85-89, 2006.

[11] C. Stutz and T. A. Runkler, "Classification and prediction of road traffic using application-specific fuzzy clustering," IEEE Transactions on Fuzzy Systems, vol. 10, no. 3, pp. 297-308, 2002.

[12] G.-Y. Jiang, J.-F. Wang, X.-D. Zhang, and L.-H. Gang, "The study on the application of fuzzy clustering analysis in the dynamic identification of road traffic state," in Proceedings of the 
2003 IEEE International Conference on Intelligent Transportation Systems, ITSC 2003, pp. 1149-1152, October 2003.

[13] A. Rizzi, A. Iacovazzi, A. Baiocchi, and S. Colabrese, "A low complexity real-time Internet traffic flows neuro-fuzzy classifier," Computer Networks, vol. 91, pp. 752-771, 2015.

[14] H. F. Guo, G. Y. Jiang, and W. G. Zhu, "Study on identification method for urban road traffic conditions with inductive loop data," in Proceedings of the 2007 IEEE International Conference on Automation and Logistics, ICAL 2007, pp. 1736-1740, August 2007.

[15] S. L. He, J. Zhang, Y. Cheng, X. Wan, and B. Ran, "Freeway Multisensor Data Fusion Approach Integrating Data from Cellphone Probes and Fixed Sensors," Journal of Sensors, vol. 2016, Article ID 7269382, 2016.

[16] C. Backfrieder, G. Ostermayer, and C. F. Mecklenbrauker, "Increased traffic flow through node-based bottleneck prediction and V2X communication," IEEE Transactions on Intelligent Transportation Systems, vol. 18, no. 2, pp. 349-363, 2017.

[17] B. Schünemann, "V2X simulation runtime infrastructure VSimRTI: An assessment tool to design smart traffic management systems," Computer Networks, vol. 55, no. 14, pp. 31893198, 2011.

[18] S. Otsuki and H. Miwa, "Contents Delivery Method Using Route Prediction in Traffic Offloading by V2X," in Proceedings of the 7th International Conference on Intelligent Networking and Collaborative Systems, IEEE INCoS 2015, pp. 239-245, twn, September 2015.

[19] J. W. Wedel, B. Schünemann, and I. Radusch, "V2X-based traffic congestion recognition and avoidance," in Proceedings of the 10th International Symposium on Pervasive Systems, Algorithms, and Networks (SPAN '09), pp. 637-641, IEEE, Kaohsiung, Taiwan, December 2009.

[20] C. Ding, S. Mishra, G. Q. Lu, J. W. Yang, and C. Liu, "Influences of built environment characteristics and individual factors on commuting distance: a multilevel mixture hazard modeling approach," Transportation Research Part D: Transport and Environment, vol. 52, pp. 314-325, 2017.

[21] C. Ding, C. Liu, Y. Zhang, J. W. Yang, and Y. P. Wang, "Investigating the impacts of built environment on vehicle miles traveled and energy consumption: Differences between commuting and non-commuting trips," Cities, vol. 68, pp. 2536, 2017. 


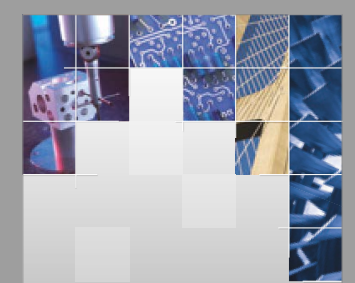

\section{Enfincering}
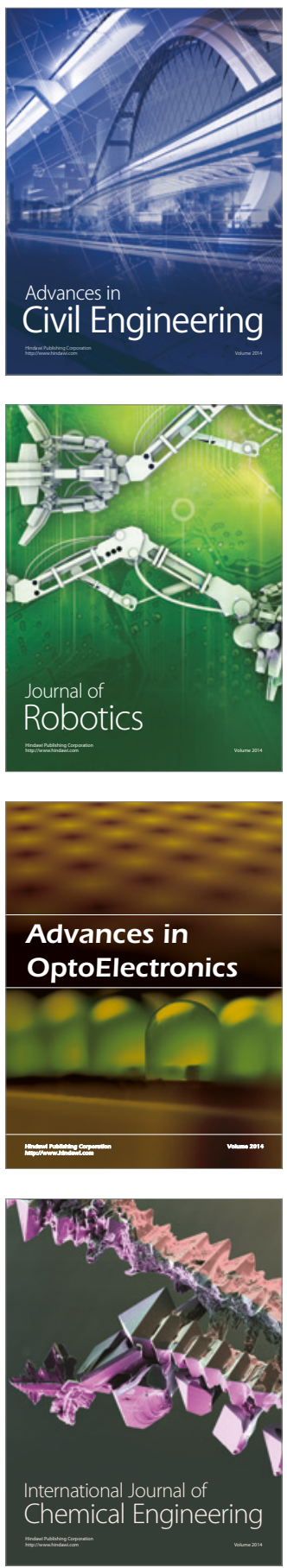

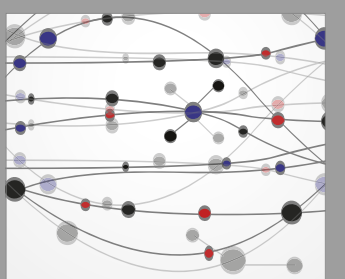

The Scientific World Journal

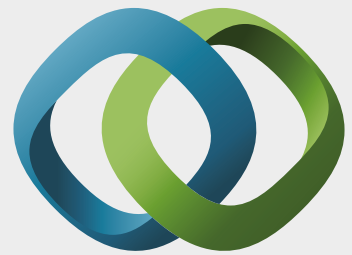

\section{Hindawi}

Submit your manuscripts at

https://www.hindawi.com
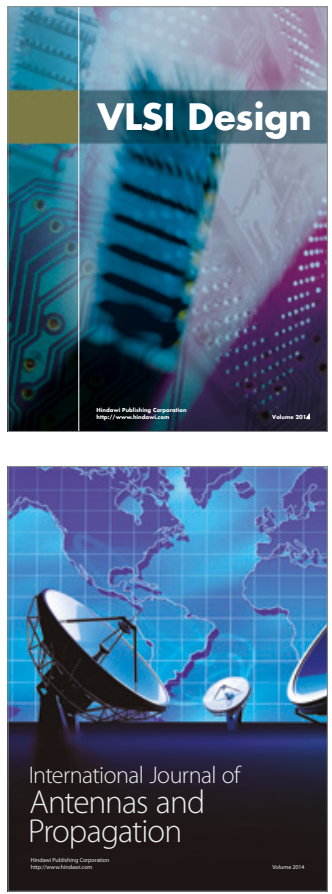

\section{Rotating}

Machinery
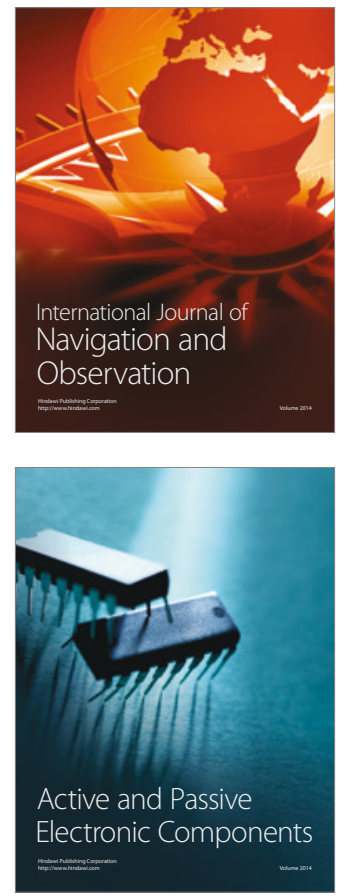
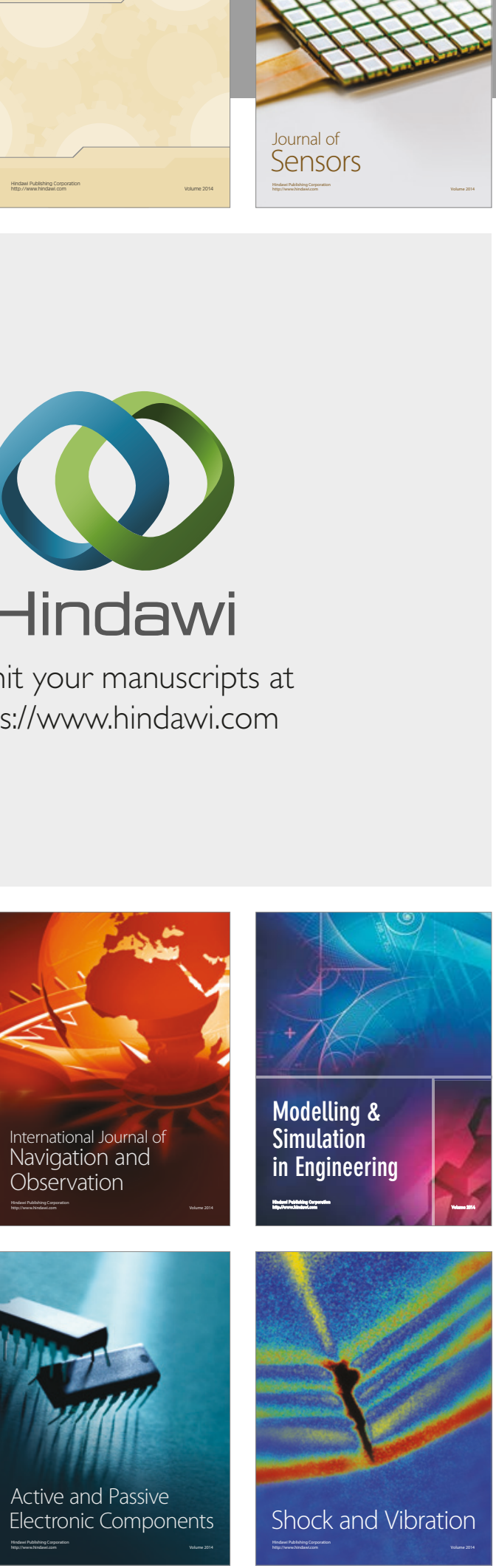
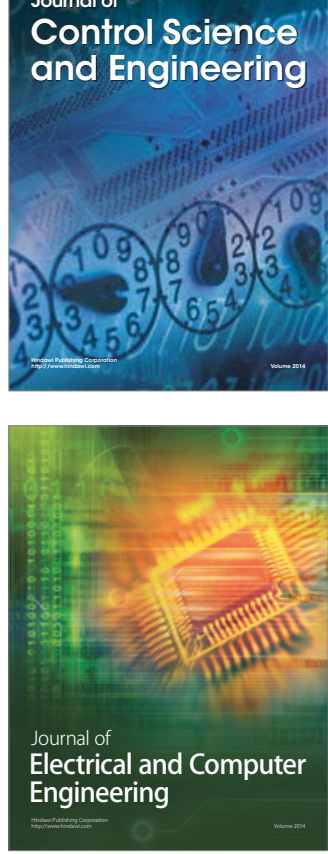

Distributed

Journal of

Control Science

and Engineering
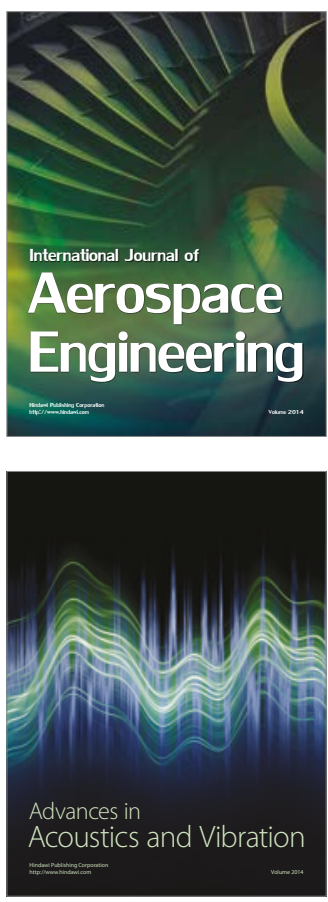

Sensor Networks 